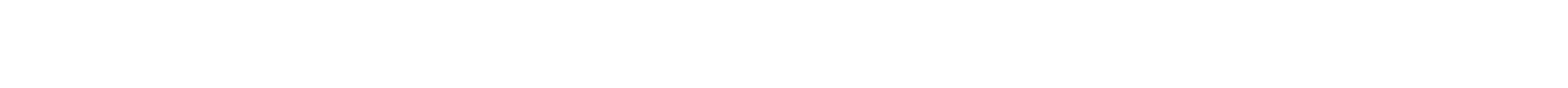 اللففلي المضادات البيتا -لاكتلم
}

\author{
صبهي aسين خلف \\ كلية التمريض \\ جلمعة الموصل
}

محمود زكي الهيلو

قمم الفيزياء الحياتية

كلية العلوم

جلمعة الموصل

(تاريخ المستلام 19 / 2 / 2013 ؛ تاريخ القبول 29 / 4 / 2013)

\section{الملغص}

عزلت (104) عزلة من العصيت المعوية للسالبة لصبغة كرالم من مجموع (700) عينة قشع ملخخونة من إصابت الجهاز التفني المفلي، وبنسبة عزل (14.8\%)، وكانت العزلات تابعة لأنواع Pseudomonas Enterobacter aerogenes, Escherichia coli, Klebsiella pneumoniae, aeruginosa Serratia marcescens, Proteus mirabilis, Enterobacter cloacae, لُألرت العزلات مقاومة واضحة لمضادات البيتا -لكتلم بأنواعها المختلفة بما فيهاسيفالوسبورينات الجل الثالث، حيث بلغت نسب المقاومة لمضادات: Cefixime، Cefotaxime، (52.9\%)،

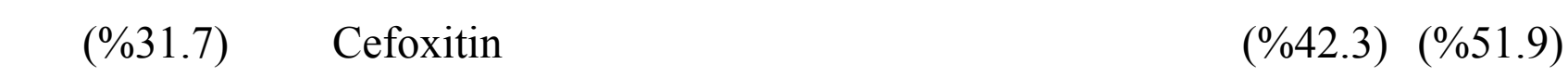

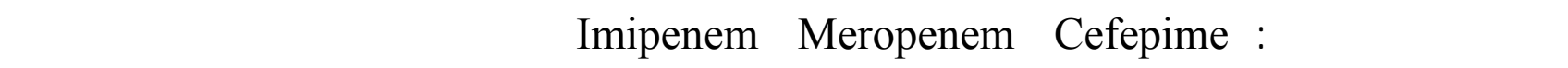
Ps. انغضضت نسب المقاومة لها لتبلغ (8.7\%)، (1.9\%)، (1.0\%) على التوالي. وكانت عزلات جرثالية aeruginosa

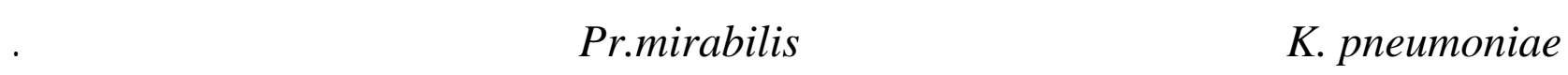

الكاملت الدالة: مقاومة العصيلت للسالبة الكرلم لمضادات البيتا -لاكتل . 


\title{
Resistance of some Gram Negative Enteric Bacilli Isolated from Lower Respiratory Tract Infections to $\beta$-lactam Antibiotics
}

\author{
Mahmood Z. Al-Hasso \\ Department of Biophysics \\ College of Science \\ University of Mosul
}

\author{
Subhi H. Khalaf \\ College of Nursing \\ University of Mosul
}

\begin{abstract}
One hundred and four isolates of gram negative enteric bacilli were isolated from (700) sputum specimens taken from lower respiratory tract infections with isolation percentage of $(14.8 \%)$. The bacterial isolates belonged to the species: Pseudomonas aeruginosa, Klebsiella pneumoniae, Escherichia coli, Enterobacter aerogenes, Enterobacter cloacae, Serratia marcescens, Proteus mirabilis, with variable isolation percentages. The isolates showed an obvious resistance to different $\beta$-lactam antibiotics including third generation cephalosporins, where the resistance percentages to Cefixime, Cefotaxime, Ceftazidime reached $(52.9 \%),(51.9 \%),(42.3 \%)$ respectively, they also showed resistance to Cefoxitin (31.7\%), while Cefepime, Meropenem, Imipenem had a high activity against the isolates, as the resistance rates were reduced to reach $(8.7 \%),(1.9 \%),(1.0 \%)$ respectively. Ps.aeruginosa isolates were the most resistant species to $\beta$-lactams, followed by K.pneumoniae isolates, while Pr. mirabilis isolates were the most susceptible ones.
\end{abstract}

Keywords: Resistance of Gram negative bacilli to $\beta$-lactam antimicrobials.

\section{الفمدة}

تعد العصيلت المعوية للسالبة لصبغة كرلم ممثلةً بأفراد العائلة المعوية وجرثومة Pseudomonas aeruginosa

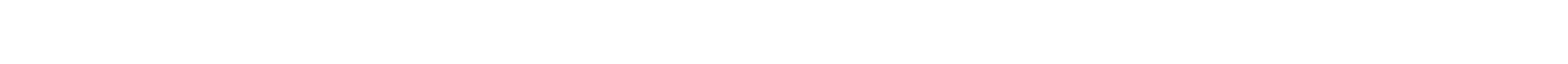

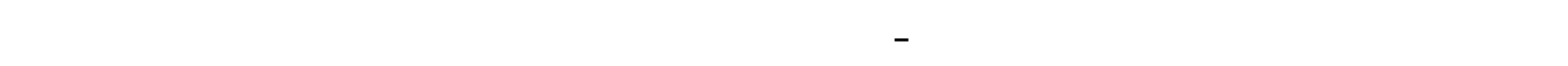

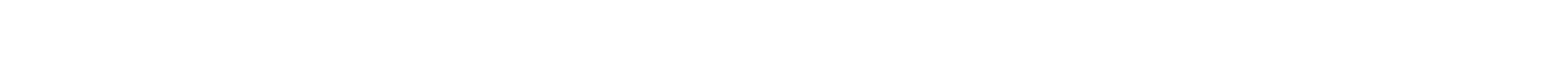
(Orrett, 2004 ; Wichard et al., 2005 ; Choi et al., 2008 ; Jean et al., 2009) مضادات البيتا -لاكتلم واللستخدام العثولئي لها في كثير من الأحيلنساعد في النتخلب السلالات الجرثومية

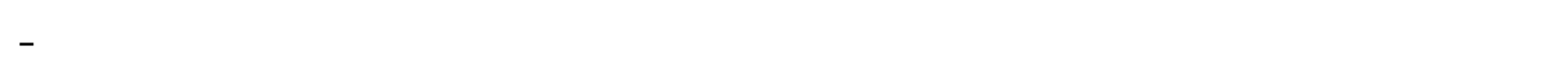

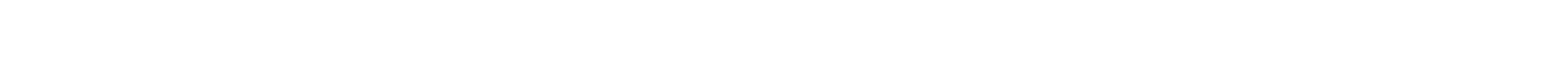

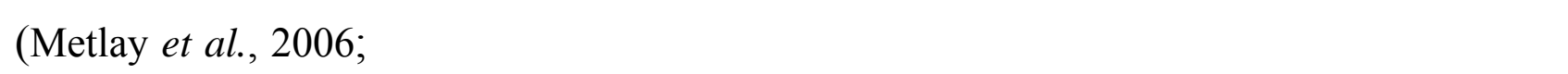
.Varaiya et al., 2008; Jean et al., 2009 ; Aminizadeh and Kashi, 2011) 
تقاوم الجراثيم المختلفة مضادات البيتا -لاكتلم بآليت متباينة ومتعدة إلا أن أبرزها ولكثرها أهميةً

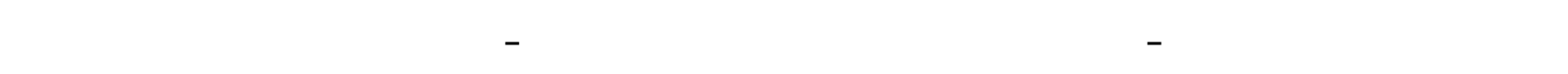
وكذك غيلب بعض المسقبلات الخلوية لمضادات البيتا -لاكتلم وهي البروتينات الرلبة للبنسلينالين (PBPs)،

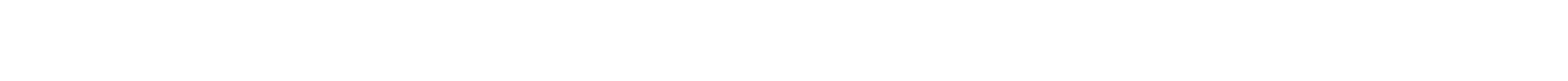

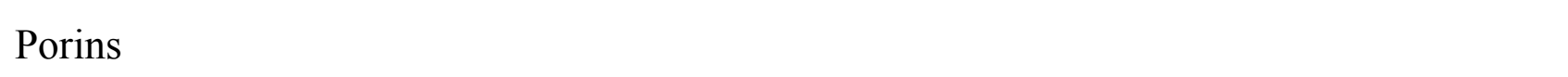
(Bononi et al., 2008; Varaiya et al., 2008; Aminizadeh and Kashi, 2011) الحالية بهرف التعرف على نسب مسلسية ومقاومة العصيت المعوية للسالبة لصبغة كرلم المعزولة من

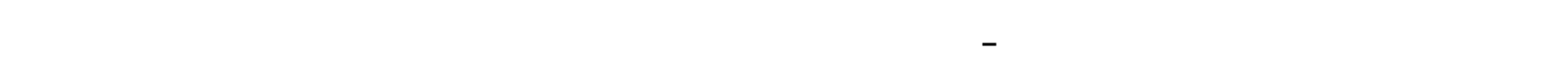

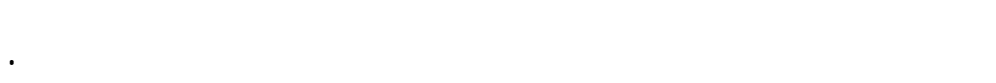

\section{المواد ولرائق الهلى}

الهزل والتشخيص

جمعت (700) عينة قثع من إصابت الجهاز التفسي اللسفلي من المرضى الوافين إلى مركز مكلفحة التدرن والأمراض الصدرية في مدينة الموصل. وضعت العينت في عبوات زجلجية ظيفة ومعقمة وققلت

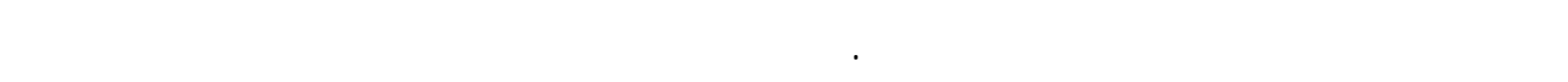

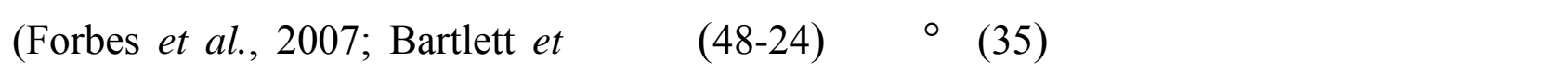
(al.,1998. قم تقية الجراثيم المخمرة وغير المخمرة للكتوز وشخصت اعتماداً على صفاتنها الفظهرية والكيموحيوية (Mac Faddin,1985; Forbes et al., 2007; Winn et al., 2006).

\section{الختبار الهسلسية لامضادك الحيوبة جلرقة الانتشار بالفأفراص}

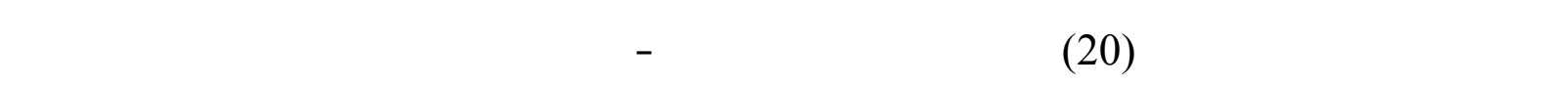

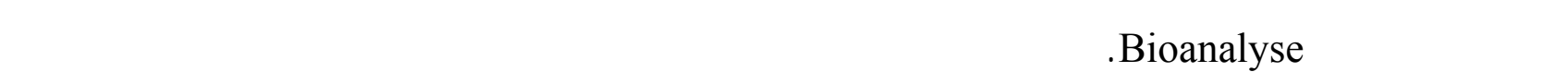
- بلستخدلم ويط لكار مولر - National Committee for Clinical Laboratory Standards (NCCLS) هنتون. حهفر معلق من الجراثيم الفتية مكافئ في عكارته للأنبوب رقم (0.5) من أنابيب ملكفرلاند القيلسية

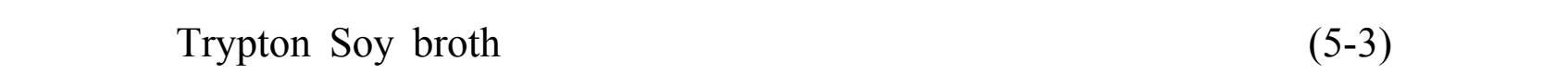

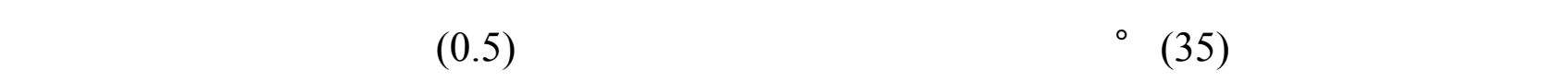

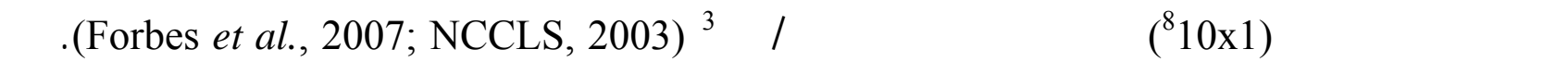

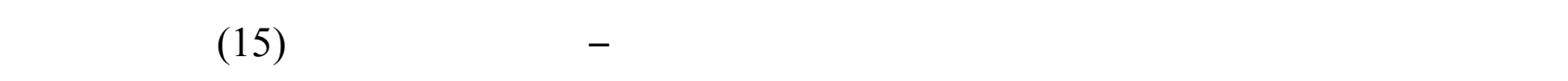

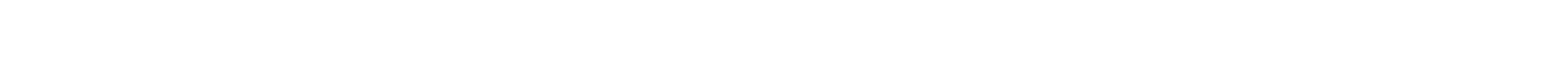

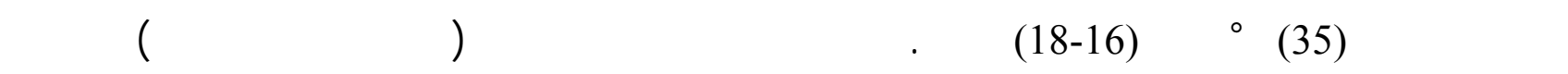


العزلات إلى ثلاث فئلت هي الهسلسة، والمتوسطة الحسلسية، والمقاومة اعتماداً على القيلست العالمية لل ـ .(NCCLS, 2003, 2004 ; Schwalbe et al., 2007) NCCLS

\section{النتائج و المنالنشة}

جمعت (700) عينة قشع من إصابت الجهاز التفهي للسفلي عزلت منها (104) عزلة من العصيت

اللسالبة لصبغة كرلم التابعة للعائلة المعوية وللنوع Ps.aeruginosa وبنسبة (14.8\%). ويبين الجدول (1) الأنواع الجرثومية المعزولة ونسب عزلها.

الجطل 1: النب المئوبة لهز المصيلت المعوبة من إصلبلت الجهاز النفس اللفلي

\begin{tabular}{|c|c|}
\hline المد (\%) & النوع الجرثوي \\
\hline$(3.7) 26$ & Pseudomonas aeruginosa \\
\hline (3.6) 25 & Klebsiella pneumoniae \\
\hline$(4.0) 28$ & Escherichia coli \\
\hline$(1.1) 8$ & Enterobacter aerogenes \\
\hline$(1.1) 8$ & Serratia marcescens \\
\hline$(0.7) 5$ & Enterobacter cloacae \\
\hline$(0.6) 4$ & Proteus mirabilis \\
\hline$(14.8) 104$ & Eوالمجالم \\
\hline
\end{tabular}

تمل هذه الجراثيم ممرضلت النتهازية تسبب الإصابت في حالات الضعف المناعي أو عند وصولها إلى منطق حسلسة من الجهم، ومما يزيدمن خطورة الإصابك التي تسببها هذه الجراثيم هومقاومتها العالية

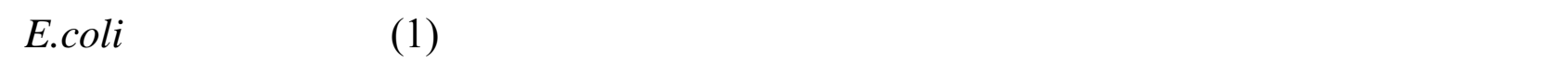
(K.pneumoniae، Ps.aeruginosa

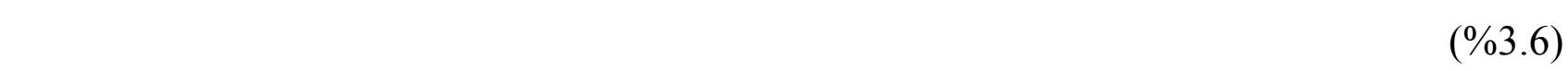

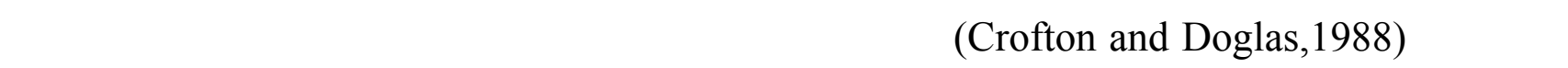

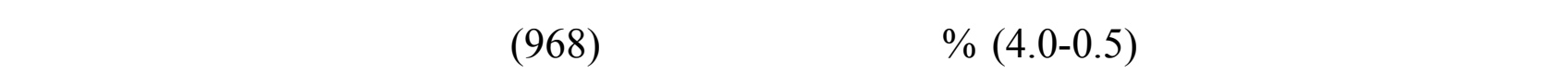
تكون شدية الططورة ومهدة للحيلة مل الإصابك الناتجة عن جرثومة (K.pneumoniae، للاسيما المكتبة aن المستشفيت (Saeed et al., 2009)، كما تقفق هذه النتائج مع ما نكره البلحثون في أن جراثيم أثشبل القولونيات عادة ما تسبب (Bartlett et al.,1998; Aminizadeh and Kashi, 2011) نسبة قليلة من إصابك الجهاز التفسي للسفلي وخاصة ذات الرئة، وان هذه النبة تكون أقل من (1\%) في 
بعض الأحيان إلا أنها تؤخذ بعين الأهمية ظارَ لكونها غالباً ما تكون مقاومة للعلاج بالمضادات الحيوية بسب لمتلكها لآليت المقاومة المختلفة للاسيما ألزيمات البيتا -لاكتلميز.

\section{الختبار الهسلسية للمضادك الحيوبة جلرقة الانتشار بالفراص}

تبين الجداول (2-9) النتائج التي مت الحصول عليها في البهث الحالي، إذ لمتخدم (20) نوعاَ من

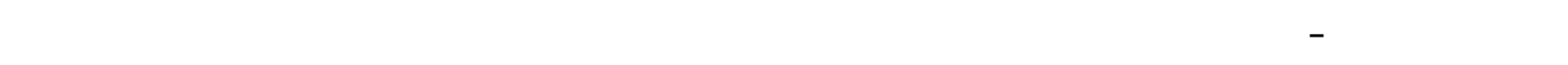
Monobactams وال ـ Carbapenems. ويبين الجدول (2) نتيجة هذا الختبار ضد العزلات الجرثومية قيد الدرلسة بصورة علمة، حيث لُٔلهرت العزلات مقاومة عالية لمضادات: Cephalothin, Ampicillin Amoxicillin, للعائلة المعوية وكذلكسلالات النوع Ps.aeruginosa غالباً ما قهر مقاومة عالية جداً لمضادات البيتا -

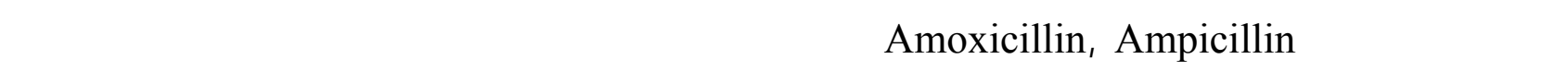

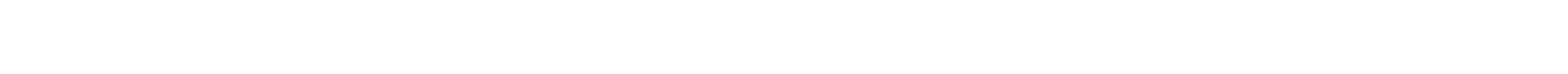

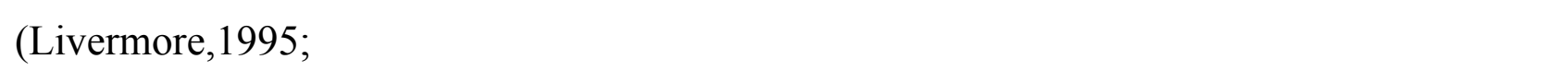
(Bradford, 2001; Khan et al., 2008; Saeed et al., 2009) بلغت (34.6) Cefdinir, Carbencillin, Ceftriaxone, Cefoperazone, Cefoxitin, Cefpodoxime (31.7\%)، (29.8\%)، (27.9\%)، (24.0\%)، (23.1\%) على التوالي، وهي بذك تشير إلى المقاومة العالية

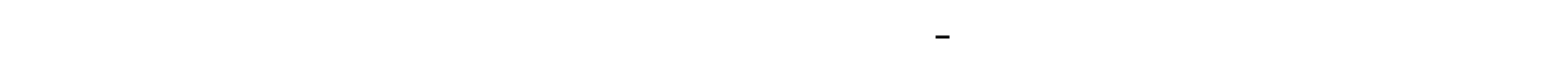

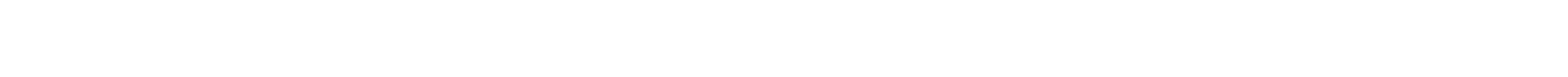

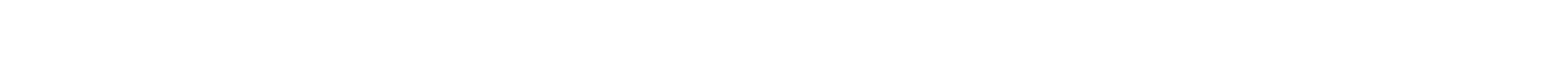

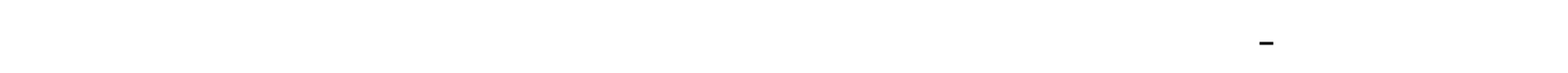

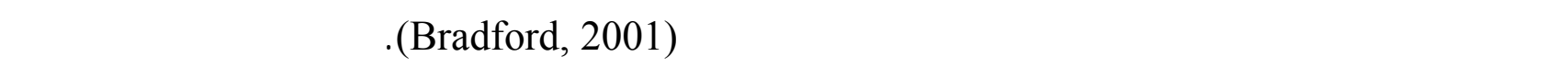

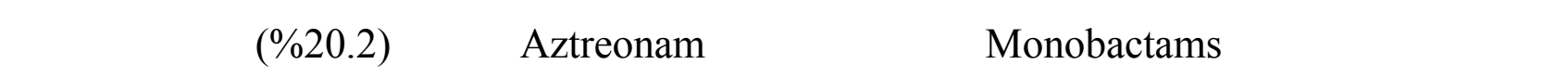

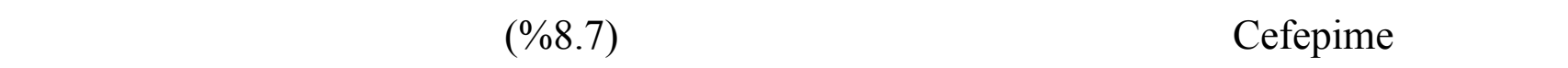

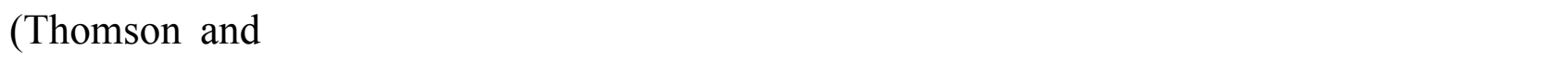
(من فoland, 2001)

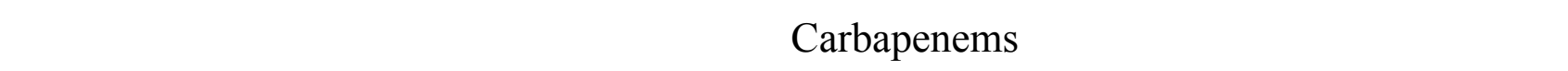

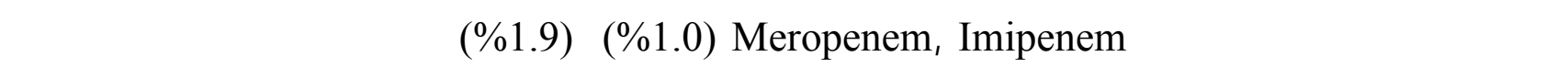
ألثارت إليه العديدمن البحوث حول فعالية هذه المضادات ضد العزلات المقاومة بمختلف أنواعها وهذا ربما

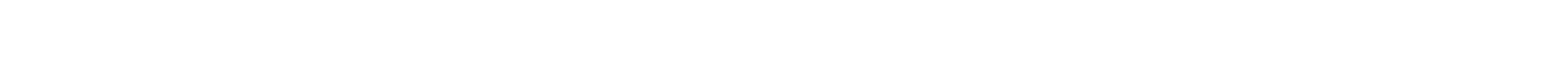


بالإضافة إلى ثباتيتها العالية تجه مظم أنزيمكت البيتا -لكتلميز رغم أن هنك مجموعة من الأنزيملت القادرة

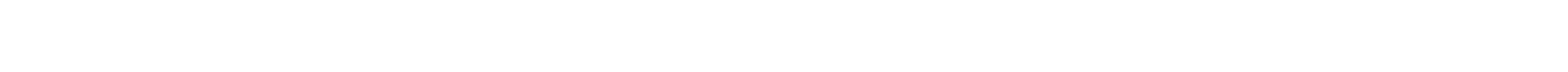

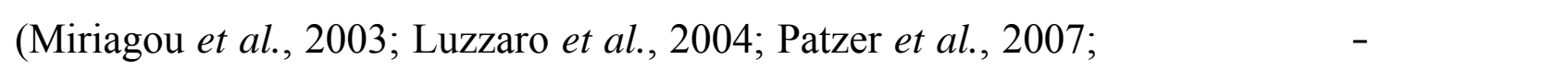
ويتضح دور حلمض الكلافيولين في تشبط أنزيملت البيتا -لاكتلميز من ملاخظة نسبة .Jean et al., 2009) المقاومة لمضاد Amoxicillin لوحله التي بلغت (85.6\%) في حن أنها انغخفضت إلى (51.0\%) في في حالة Amoxicillin-Clavulinic Acid

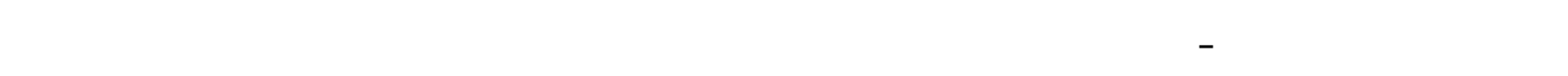

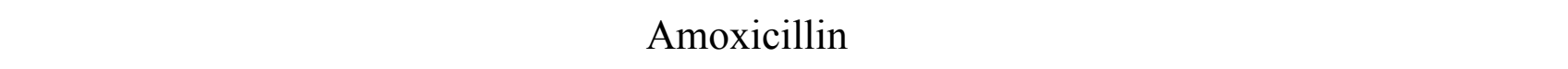

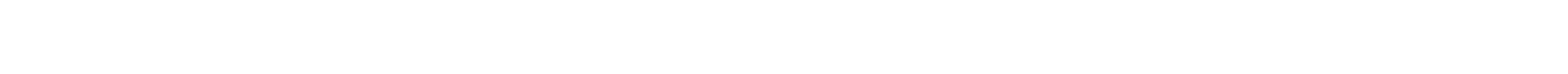

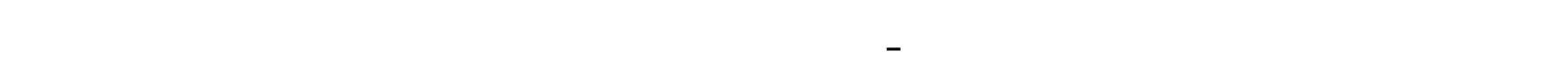

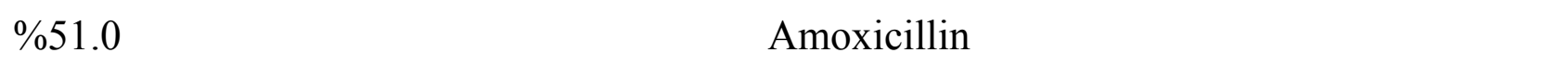

.(Blazquez et al.,1993)

وتبين الجداول (3-9-9) الهسلسية لمضادات البيتا -لاكتلم هسب النوع الجرثومي، حيث كافت عزلات

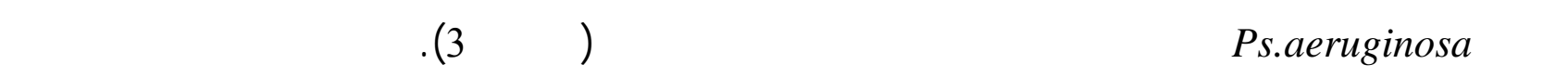

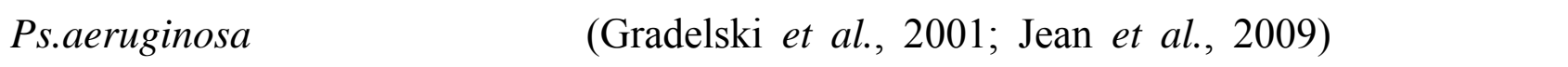

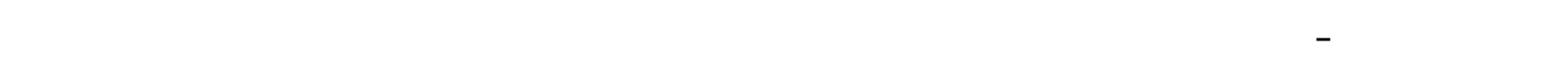
Carbapenems, Cephamycins, Monobactams

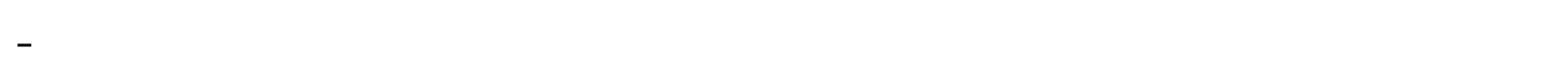

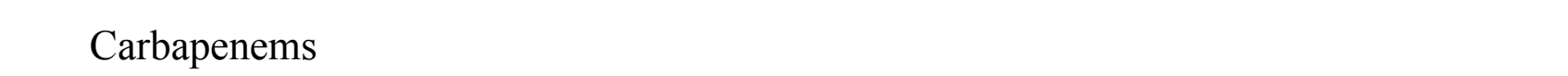
أنها تتميز بفانيتها المنغضضة لعدد من المضادات الحيوية نتيجة اففقارها لبورينات غثائئية معينة أو تحويرها

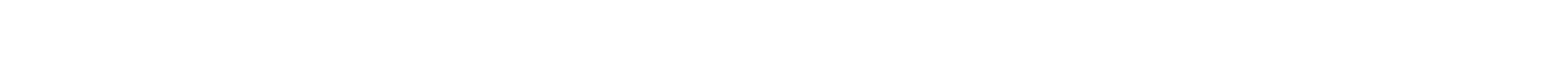

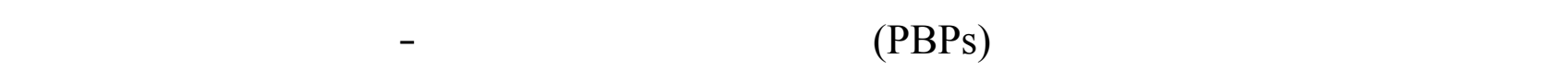

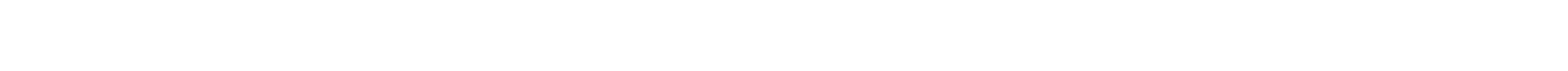
.(Khan et al., 2008; Varaiya et al., 2008; Dugal and Fernandes, 2011) 


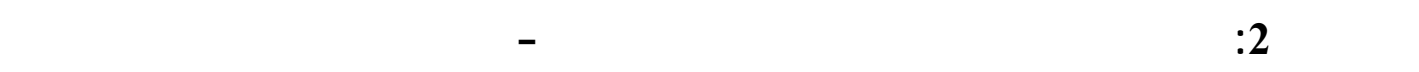

\begin{tabular}{|c|c|c|c|c|c|}
\hline $\begin{array}{c}\text { المقاومة العدد } \\
\text { (\%) }\end{array}$ & 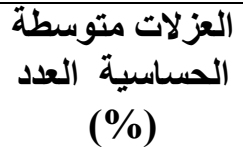 & العزلات الحساسة (\%) & (مايكروغرام/قرص) التركيز & الرمز & المضاد الحيوي \\
\hline $\begin{array}{l}(93.3) 97 \\
\end{array}$ & (4.8) 5 & (1.9) 2 & 10 & AM & Ampicillin \\
\hline (85.6) 89 & (10.6) 11 & $(3.8) 4$ & 30 & KF & Cephalothin \\
\hline (85.6) 89 & $(2.9) 3$ & $(11.5) 12$ & 25 & $\mathrm{AX}$ & Amoxicillin \\
\hline (76) 79 & $(12.5) 13$ & $(11.5) 12$ & 30 & $\mathrm{CL}$ & Cephalexin \\
\hline (74) 77 & $(7.7) 8$ & (18.3) 19 & 30 & CXM & Cefuroxime \\
\hline (52.9) 55 & $(11.5) 12$ & (35.6) 37 & 5 & CFM & Cefixime \\
\hline (51.9) 54 & $(8.7) 9$ & (39.4) 41 & 30 & CTX & Cefotaxime \\
\hline (51) 53 & (7.7) 8 & $(41.3) 43$ & $20 \backslash 10$ & AMC & $\begin{array}{l}\text { Amoxicillin- } \\
\text { Clavulinic acid }\end{array}$ \\
\hline$(42.3) 44$ & $(12.5) 13$ & $(45.2) 47$ & 30 & CAZ & Ceftazidime \\
\hline (34.6) 36 & $(4.8) 5$ & (60.6) 63 & 10 & CPD & Cefpodoxime \\
\hline (31.7) 33 & (3.9) 4 & $(64.4) 67$ & 30 & FOX & Cefoxitin \\
\hline$(29.8) 31$ & $(5.8) 6$ & (64.4) 67 & 75 & CEP & Cefoperazone \\
\hline$(27.9) 29$ & $(2.9) 3$ & $(69.2) 72$ & 30 & $\mathrm{CRO}$ & Ceftriaxone \\
\hline (24) 25 & $(4.8) 5$ & $(71.2) 74$ & 100 & PY & Carbencillin \\
\hline$(23.1) 24$ & $(4.8) 5$ & $(72.1) 75$ & 5 & CDR & Cefdinir \\
\hline (20.2) 21 & $(4.8) 5$ & (75) 78 & 30 & ATM & Aztreonam \\
\hline$(19.2) 20$ & $(8.7) 9$ & $(72.1) 75$ & 30 & ZOX & Ceftizoxime \\
\hline$(8.7) 9$ & $(2.9) 3$ & (88.4) 92 & 30 & FEP & Cefepime \\
\hline (1.9) 2 & $(2.9) 3$ & (95.2) 99 & 10 & MEM & Meropenem \\
\hline (1) 1 & (0) 0 & (99) 103 & 10 & IPM & Imipenem \\
\hline
\end{tabular}

\section{الجطل 3 : مسلسية عزلات جرثومة Ps.aeruginosa لمضادك البيتا للكتلم جلرقة الانتشار بلأفرلص}

\begin{tabular}{|c|c|c|c|c|c|}
\hline العزلات المقاومة العدد & 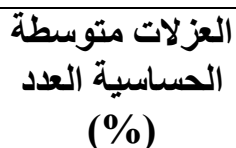 & العزلات الحساسة (\%) & 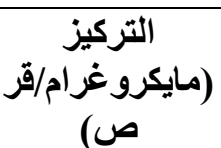 & الرمز & المضاد الحيوي \\
\hline (100) 26 & (0) 0 & (0) 0 & 10 & $\mathrm{AM}$ & Ampicillin \\
\hline$(96.15) 25$ & $(3.85) 1$ & (0) 0 & 30 & $\mathrm{KF}$ & Cephalothin \\
\hline (100) 26 & (0) 0 & (0) 0 & 25 & $\mathrm{AX}$ & Amoxicillin \\
\hline$(80.75) 21$ & (15.4) 4 & $(3.85) 1$ & 30 & $\mathrm{CL}$ & Cephalexin \\
\hline$(92.3) 24$ & (0) 0 & (7.7) 2 & 30 & CXM & Cefuroxime \\
\hline (73.1) 19 & (7.7) 2 & (19.2) 5 & 5 & CFM & Cefixime \\
\hline$(53.85) 14$ & $(3.85) 1$ & $(42.3) 11$ & 30 & CTX & Cefotaxime \\
\hline$(69.25) 18$ & $(11.55) 3$ & $(19.2) 5$ & $20 \backslash 10$ & AMC & $\begin{array}{c}\text { Amoxicillin- } \\
\text { Clavulinic acid }\end{array}$ \\
\hline (34.6) 9 & $(11.55) 3$ & $(53.85) 14$ & 30 & CAZ & Ceftazidime \\
\hline (34.6) 9 & (7.7) 2 & $(57.7) 15$ & 10 & CPD & Cefpodoxime \\
\hline$(61.55) 16$ & $(11.55) 3$ & (26.9) 7 & 30 & FOX & Cefoxitin \\
\hline$(11.55) 3$ & $(7.7) 2$ & $(80.75) 21$ & 75 & CEP & Cefoperazone \\
\hline$(38.5) 10$ & (0) 0 & $(61.5) 16$ & 30 & $\mathrm{CRO}$ & Ceftriaxone \\
\hline (26.9) 7 & $(11.55) 3$ & $(61.55) 16$ & 100 & PY & Carbencillin \\
\hline$(19.2) 5$ & $(3.85) 1$ & $(76.95) 20$ & 5 & CDR & Cefdinir \\
\hline$(26.9) 7$ & $(3.85) 1$ & $(69.25) 18$ & 30 & ATM & Aztreonam \\
\hline (19.2) 5 & (15.4) 4 & $(65.4) 17$ & 30 & ZOX & Ceftizoxime \\
\hline (15.4) 4 & $(7.7) 2$ & $(76.9)$ & 30 & FEP & Cefepime \\
\hline$(7.7) 2$ & $(11.55) 3$ & $(80.75) 21$ & 10 & MEM & Meropenem \\
\hline$(3.85) 1$ & (0) 0 & $(96.15) 25$ & 10 & IPM & Imipenem \\
\hline
\end{tabular}




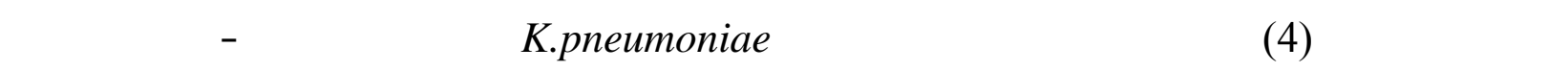

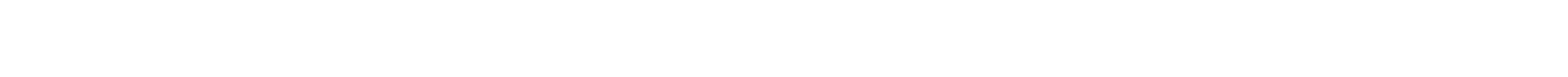

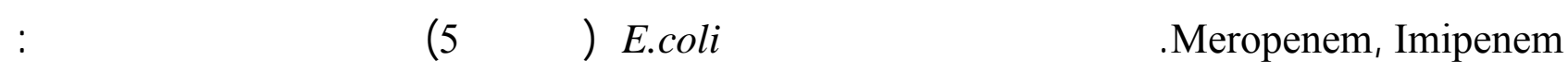
Cefotaxime Amoxicillin, Cephalothin, Ampicillin

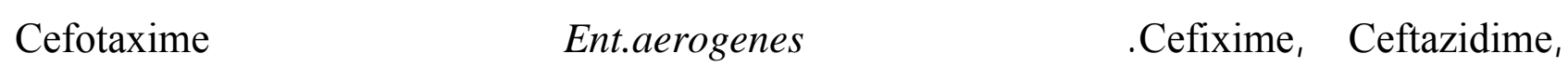
Cefixime, Ceftazidime, (25.0) Cefpodoxime, Carbencillin, Cefdinir, Cefoxitin, Ceftriaxone العزلات عالية المسلسية لمضادات Meropenem, Imipenem, Cefepime (الجدول 6). أما عزلات جرثومة S.marcescens فكانت مقاومة لمضاديّ Amoxicillin, Ampicillin بنسبة (100\%) لكل منهما ولمضادات Cefuroxime, Cephalexin, Cephalothin بنسبة (75.0\%) لكل منها، في حين لظعهرت المقاومة لمضادات Ceftazidime, Ceftriaxone, Cefixime بنسبة (25.0\%) لكل منها، في حني كلت فين العزلات جسلسة بشكل مطلق لمضادات Meropenem, Imipenem, Cefepime, Cefoxitin (الجدول 7).

الجطر 4 : مسلسية عزلات جرثومة K. pneumoniae المضادل البيتا -لكتله برقة النتشار بلأفراه

\begin{tabular}{|c|c|c|c|c|c|}
\hline 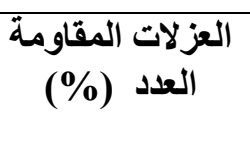 & 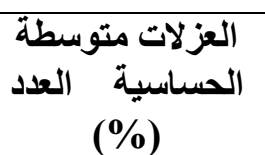 & العزلات الحساسة (\%) & 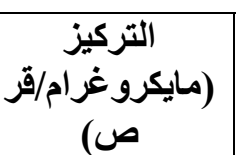 & الرمز & المضاد الحيوي \\
\hline 25 & (0) 0 & (0) 0 & 10 & AM & Ampicillin \\
\hline (92) 23 & (8) 2 & (0) 0 & 30 & KF & Cephalothin \\
\hline (80) 20 & (8) 2 & (12) 3 & 25 & $\mathrm{AX}$ & Amoxicillin \\
\hline (80) 20 & (8) 2 & (12) 3 & 30 & $\mathrm{CL}$ & Cephalexin \\
\hline (76) 19 & (8) 2 & (16) 4 & 30 & CXM & Cefuroxime \\
\hline (60) 15 & (20) 5 & (20) 5 & 5 & CFM & Cefixime \\
\hline (72) 18 & (12) 3 & (16) 4 & 30 & CTX & Cefotaxime \\
\hline (52) 13 & (8) 2 & (40) 10 & $20 \backslash 10$ & $\mathrm{AMC}$ & $\begin{array}{c}\text { Amoxicillin- } \\
\text { Clavulinic acid }\end{array}$ \\
\hline (60) 15 & (16) 4 & $(24) 6$ & 30 & CAZ & Ceftazidime \\
\hline (64) 16 & (4) 1 & $(32) 8$ & 10 & CPD & Cefpodoxime \\
\hline (36) 9 & (0) 0 & (64) 16 & 30 & FOX & Cefoxitin \\
\hline (56) 14 & (8) 2 & (36) 9 & 75 & CEP & Cefoperazone \\
\hline$(28) 7$ & (0) 0 & (72) 18 & 30 & $\mathrm{CRO}$ & Ceftriaxone \\
\hline (24) 6 & (4) 1 & (72) 18 & 100 & PY & Carbencillin \\
\hline (36) 9 & (4) 1 & (60) 15 & 5 & CDR & Cefdinir \\
\hline (12) 3 & (8) 2 & $(80) 20$ & 30 & ATM & Aztreonam \\
\hline (32) 8 & (8) 2 & (60) 15 & 30 & $\mathrm{ZOX}$ & Ceftizoxime \\
\hline$(8) 2$ & (0) 0 & (92) 23 & 30 & FEP & Cefepime \\
\hline (0) 0 & (0) 0 & $(100) 25$ & 10 & MEM & Meropenem \\
\hline (0) 0 & (0) 0 & (100) 25 & 10 & IPM & Imipenem \\
\hline
\end{tabular}


مقاومة بعض العصيت المعوية للسالبة.

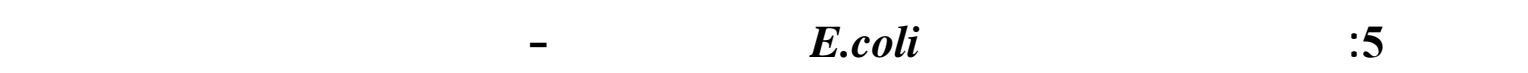

\begin{tabular}{|c|c|c|c|c|c|}
\hline العزلات المقاومة & العزلات متوسطة & العزلات الحساسة & التركيز & & \\
\hline $\begin{array}{l}\text { العدد } \\
\text { (\%) }\end{array}$ & 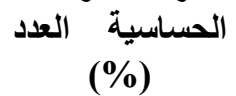 & $\begin{array}{l}\text { العدد } \\
\text { (\%) }\end{array}$ & (مايكروغرام/قرص) & 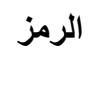 & المضاد الحيوي \\
\hline$(85.7) 24$ & $(7.15) 2$ & $(7.15) 2$ & 10 & $\mathrm{AM}$ & Ampicillin \\
\hline$(82.15) 23$ & (10.7) 3 & $(7.15) 2$ & 30 & KF & Cephalothin \\
\hline$(78.5) 22$ & (3.6) 1 & (17.9) 5 & 25 & $\mathrm{AX}$ & Amoxicillin \\
\hline (71.4) 20 & (10.7) 3 & (17.9) 5 & 30 & CL & Cephalexin \\
\hline$(57.15) 16$ & $(10.7) 3$ & $(32.15) 9$ & 30 & CXM & Cefuroxime \\
\hline$(50) 14$ & $(7.15) 2$ & $(42.85) 12$ & 5 & CFM & Cefixime \\
\hline$(57.15) 16$ & $(7.15) 2$ & $(35.7) 10$ & 30 & CTX & Cefotaxime \\
\hline$(39.25) 11$ & (3.6) 1 & $(57.15) 16$ & $20 \backslash 10$ & AMC & $\begin{array}{c}\text { Amoxicillin- } \\
\text { Clavulinic acid }\end{array}$ \\
\hline$(46.45) 13$ & $(10.7) 3$ & $(42.85) 12$ & 30 & CAZ & Ceftazidime \\
\hline$(28.6) 8$ & (3.6) 1 & $(67.8) 19$ & 10 & $\mathrm{CPD}$ & Cefpodoxime \\
\hline (17.9) 5 & (3.6) 1 & $(78.5) 22$ & 30 & FOX & Cefoxitin \\
\hline (21.4) 6 & (3.6) 1 & $(75) 21$ & 75 & CEP & Cefoperazone \\
\hline$(25) 7$ & (3.6) 1 & (71.4) 20 & 30 & $\mathrm{CRO}$ & Ceftriaxone \\
\hline (28.6) 8 & (0) 0 & (71.4) 20 & 100 & PY & Carbencillin \\
\hline$(25) 7$ & (3.6) 1 & (71.4) 20 & 5 & CDR & Cefdinir \\
\hline$(14.25) 4$ & (3.6) 1 & $(82.15) 23$ & 30 & ATM & Aztreonam \\
\hline$(10.7) 3$ & (3.6) 1 & $(85.7) 24$ & 30 & $\mathrm{ZOX}$ & Ceftizoxime \\
\hline (3.6) 1 & (0) 0 & $(96.4) 27$ & 30 & FEP & Cefepime \\
\hline (0) 0 & (0) 0 & (100) 28 & 10 & MEM & Meropenem \\
\hline (0) 0 & (0) 0 & (100) 28 & 10 & IPM & Imipenem \\
\hline
\end{tabular}

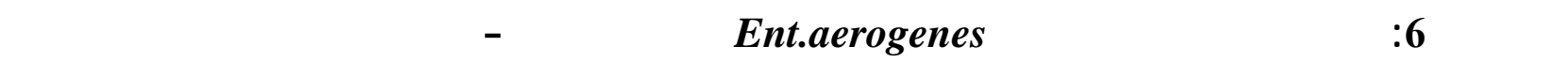

\begin{tabular}{|c|c|c|c|c|c|}
\hline $\begin{array}{c}\text { المقاومة العدد } \\
\text { (\%) }\end{array}$ & 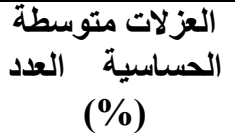 & العزلات الحساسة (\%) & 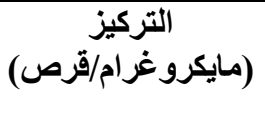 & 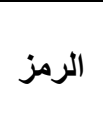 & المضاد الحيوي \\
\hline$(87.5) 7$ & $(12.5) 1$ & (0) 0 & 10 & AM & Ampicillin \\
\hline (87.5) 7 & $(12.5) 1$ & (0) 0 & 30 & KF & Cephalothin \\
\hline (75) 6 & (0) 0 & (25) 2 & 25 & AX & Amoxicillin \\
\hline (75) 6 & (25) 2 & (0) 0 & 30 & $\mathrm{CL}$ & Cephalexin \\
\hline$(87.5) 7$ & $(12.5) 1$ & (0) 0 & 30 & CXM & Cefuroxime \\
\hline (37.5) 3 & $(12.5) 1$ & (50) 4 & 5 & CFM & Cefixime \\
\hline$(37.5) 3$ & (12.5) 1 & (50) 4 & 30 & CTX & Cefotaxime \\
\hline$(50) 4$ & (12.5) 1 & $(37.5) 3$ & $20 \backslash 10$ & AMC & $\begin{array}{c}\text { Amoxicillin- } \\
\text { Clavulinic acid }\end{array}$ \\
\hline$(37.5) 3$ & $(12.5) 1$ & (50) 4 & 30 & CAZ & Ceftazidime \\
\hline$(25) 2$ & $(12.5) 1$ & $(62.5) 5$ & 10 & CPD & Cefpodoxime \\
\hline (25) 2 & (0) 0 & $(75) 6$ & 30 & FOX & Cefoxitin \\
\hline (50) 4 & $(0) 0$ & (50) 4 & 75 & CEP & Cefoperazone \\
\hline (25) 2 & $(12.5) 1$ & $(62.5) 5$ & 30 & $\mathrm{CRO}$ & Ceftriaxone \\
\hline (25) 2 & (0) 0 & $(75) 6$ & 100 & PY & Carbencillin \\
\hline$(25) 2$ & $(12.5) 1$ & $(62.5) 5$ & 5 & CDR & Cefdinir \\
\hline$(37.5) 3$ & (0) 0 & $(62.5) 5$ & 30 & ATM & Aztreonam \\
\hline$(37.5) 3$ & $(12.5) 1$ & (50) 4 & 30 & ZOX & Ceftizoxime \\
\hline (12.5) 1 & $(12.5) 1$ & $(75) 6$ & 30 & FEP & Cefepime \\
\hline (0) 0 & (0) 0 & (100) 8 & 10 & MEM & Meropenem \\
\hline (0) 0 & (0) 0 & (100) 8 & 10 & IPM & Imipenem \\
\hline
\end{tabular}


محمود زكي المَسّو و صبهي مسين خلف

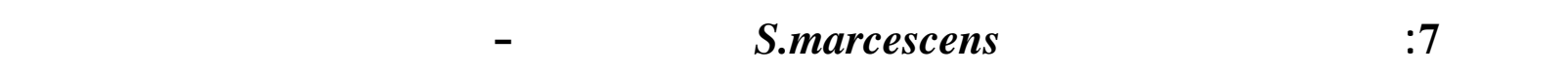

\begin{tabular}{|c|c|c|c|c|c|}
\hline 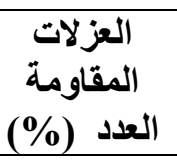 & العزلات متوسطة & 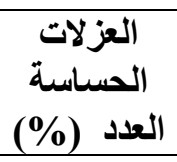 & (مايكروغرام/قرص) & الرمز & المضساد الحيوي \\
\hline$(100) 8$ & (0) 0 & (0) 0 & 10 & AM & Ampicillin \\
\hline$(75) 6$ & $(25) 2$ & (0) 0 & 30 & KF & Cephalothin \\
\hline (100) 8 & $(0) 0$ & (0) 0 & 25 & $\mathrm{AX}$ & Amoxicillin \\
\hline$(75) 6$ & $(25) 2$ & (0) 0 & 30 & $\mathrm{CL}$ & Cephalexin \\
\hline$(75) 6$ & (25) 2 & $(0) 0$ & 30 & CXM & Cefuroxime \\
\hline (25) 2 & (25) 2 & $(50) 4$ & 5 & CFM & Cefixime \\
\hline$(12.5) 1$ & $(12.5) 1$ & $(75) 6$ & 30 & CTX & Cefotaxime \\
\hline$(37.5) 3$ & $(12.5) 1$ & $(50) 4$ & $20 \backslash 10$ & $\mathrm{AMC}$ & $\begin{array}{c}\text { Amoxicillin- } \\
\text { Clavulinic acid }\end{array}$ \\
\hline$(25) 2$ & $(12.5) 1$ & $(62.5) 5$ & 30 & CAZ & Ceftazidime \\
\hline$(12.5) 1$ & $(0) 0$ & $(87.5) 7$ & 10 & CPD & Cefpodoxime \\
\hline (0) 0 & $(0) 0$ & $((100) 8$ & 30 & FOX & Cefoxitin \\
\hline (25) 2 & $(12.5) 1$ & $(62.5) 5$ & 75 & CEP & Cefoperazone \\
\hline (25) 2 & $(12.5) 1$ & $(62.5) 5$ & 30 & $\mathrm{CRO}$ & Ceftriaxone \\
\hline$(12.5) 1$ & $(12.5) 1$ & $(75) 6$ & 100 & PY & Carbencillin \\
\hline$(0) 0$ & $(12.5) 1$ & $(87.5) 7$ & 5 & CDR & Cefdinir \\
\hline$(37.5) 3$ & $(12.5) 1$ & $(50) 4$ & 30 & ATM & Aztreonam \\
\hline$(12.5) 1$ & $(12.5) 1$ & (75) 6 & 30 & $\mathrm{ZOX}$ & Ceftizoxime \\
\hline$(0) 0$ & $(0) 0$ & (100) 8 & 30 & FEP & Cefepime \\
\hline (0) 0 & (0) 0 & (100) 8 & 10 & MEM & Meropenem \\
\hline$(0) 0$ & (0) 0 & $(100) 8$ & 10 & IPM & Imipenem \\
\hline
\end{tabular}

يظهر الجدول (8)طبيعة مقاومة عزلات Ent.cloacae لمضادات البيتا -لاكتلم، حيث كلت العزلات ذات مقاومة منخفضة نسبياً لمظمسيفالوسبورينات الجل الثالث وكذك لمضاديّ فيّل

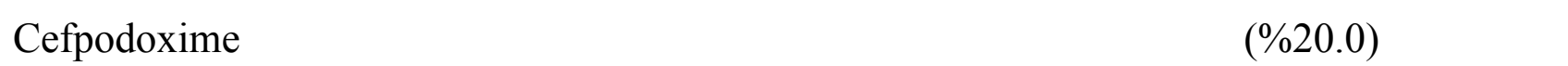
Pr.mirabilis ويبين الجدول (9) عسلسية عزلات جرثومة (Meropenem, Imipenem, Ceftizoxime, للمضادات المستخلمة في الدرلسة حيث كلتت هذه العزلات الأكثر هسلسية من بين الأنواع الجرثومية

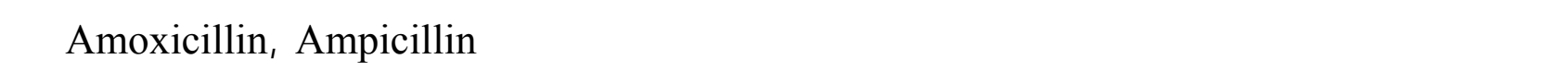
كلت مقاومة بنسبة (75.0\%) لكل منهما، كما لظٔلهرت المقاومة بنسبة (50.0\%) للك من من مضادات Amoxicillin-Clavulinic Acid, Cefuroxime, Cephalothin, Cephalexin العديد من الدرلسك من كون جرثومة Pr.mirabilis من لكثر أنواع العائلة المعوية هسلسية للمضادات (Winn et al., 2006 ; Wichard et al., 2005 ; الحيوية ما لم تكتب آليات المقاومة من جراثيم لأخرى 


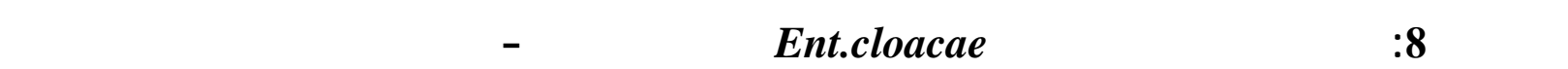

\begin{tabular}{|c|c|c|c|c|c|}
\hline 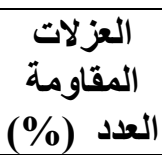 & العزلات متوسطة العدد & العزلات الحساسة (\%) & (مايكروغرام/قرص) & الرمز & المضاد الحيوي \\
\hline (80) 4 & (20) 1 & (0) 0 & 10 & AM & Ampicillin \\
\hline (60) 3 & (20) 1 & (20) 1 & 30 & KF & Cephalothin \\
\hline (80) 4 & (0) 0 & (20) 1 & 25 & $\mathrm{AX}$ & Amoxicillin \\
\hline (80) 4 & (0) 0 & (20) 1 & 30 & $\mathrm{CL}$ & Cephalexin \\
\hline (60) 3 & (0) 0 & (40) 2 & 30 & CXM & Cefuroxime \\
\hline (20) 1 & (0) 0 & (80) 4 & 5 & CFM & Cefixime \\
\hline (20) 1 & (0) 0 & (80) 4 & 30 & CTX & Cefotaxime \\
\hline (40) 2 & (0) 0 & (60) 3 & $20 \backslash 10$ & AMC & $\begin{array}{c}\text { Amoxicillin- } \\
\text { Clavulinic acid }\end{array}$ \\
\hline (20) 1 & (20) 1 & (60) 3 & 30 & CAZ & Ceftazidime \\
\hline (0) 0 & (0) 0 & (100) 5 & 10 & CPD & Cefpodoxime \\
\hline (20) 1 & (0) 0 & (80) 4 & 30 & FOX & Cefoxitin \\
\hline (40) 2 & (0) 0 & (60) 3 & 75 & CEP & Cefoperazone \\
\hline (20) 1 & (0) 0 & (80) 4 & 30 & $\mathrm{CRO}$ & Ceftriaxone \\
\hline (20) 1 & (0) 0 & (80) 4 & 100 & PY & Carbencillin \\
\hline (20) 1 & (0) 0 & (80) 4 & 5 & CDR & Cefdinir \\
\hline (20) 1 & (0) 0 & (80) 4 & 30 & ATM & Aztreonam \\
\hline (0) 0 & (0) 0 & (100) 5 & 30 & ZOX & Ceftizoxime \\
\hline (20) 1 & (0) 0 & (80) 4 & 30 & FEP & Cefepime \\
\hline (0) 0 & (0) 0 & (100) 5 & 10 & MEM & Meropenem \\
\hline (0) 0 & (0) 0 & (100) 5 & 10 & IPM & Imipenem \\
\hline
\end{tabular}

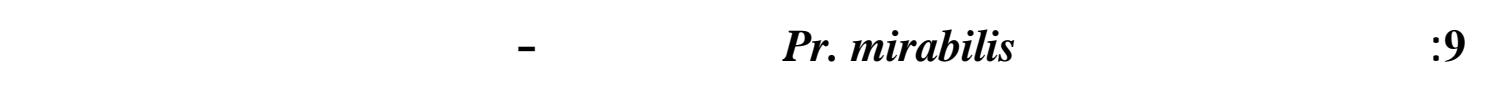

\begin{tabular}{|c|c|c|c|c|c|}
\hline العقاومة العة & 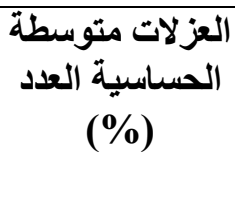 & العزلات الحساسة (\%) & (مايكروغرام/قرص) & الرمز & المضاد الحيوي \\
\hline$(75) 3$ & (25) 1 & (0) 0 & 10 & AM & Ampicillin \\
\hline (50) 2 & (25) 1 & (25) 1 & 30 & $\mathrm{KF}$ & Cephalothin \\
\hline (75) 3 & (0) 0 & (25) 1 & 25 & $\mathrm{AX}$ & Amoxicillin \\
\hline (50) 2 & (0) 0 & (50) 2 & 30 & $\mathrm{CL}$ & Cephalexin \\
\hline (50) 2 & (0) 0 & (50) 2 & 30 & CXM & Cefuroxime \\
\hline (25) 1 & (0) 0 & (75) 3 & 5 & CFM & Cefixime \\
\hline (25) 1 & (25) 1 & (50) 2 & 30 & CTX & Cefotaxime \\
\hline (50) 2 & (0) 0 & (50) 2 & $20 \backslash 10$ & AMC & $\begin{array}{c}\text { Amoxicillin- } \\
\text { Clavulinic acid }\end{array}$ \\
\hline (25) 1 & (0) 0 & (75) 3 & 30 & CAZ & Ceftazidime \\
\hline (0) 0 & (0) 0 & (100) 4 & 10 & CPD & Cefpodoxime \\
\hline (0) 0 & (0) 0 & (100) 4 & 30 & FOX & Cefoxitin \\
\hline (0) 0 & (0) 0 & (100) 4 & 75 & CEP & Cefoperazone \\
\hline (0) 0 & (0) 0 & (100) 4 & 30 & $\mathrm{CRO}$ & Ceftriaxone \\
\hline (0) 0 & (0) 0 & (100) 4 & 100 & PY & Carbencillin \\
\hline (0) 0 & (0) 0 & (100) 4 & 5 & CDR & Cefdinir \\
\hline (0) 0 & (0) 0 & (100) 4 & 30 & ATM & Aztreonam \\
\hline (0) 0 & (0) 0 & (100) 4 & 30 & ZOX & Ceftizoxime \\
\hline (0) 0 & (0) 0 & (100) 4 & 30 & FEP & Cefepime \\
\hline (0) 0 & (0) 0 & (100) 4 & 10 & MEM & Meropenem \\
\hline (0) 0 & (0) 0 & (100) 4 & 10 & IPM & Imipenem \\
\hline
\end{tabular}


إن نتائج الدرلسة الحالية تثمق مع العديد من الدرلست العالمية التي أثارت إلى مقاومة الجراثيم

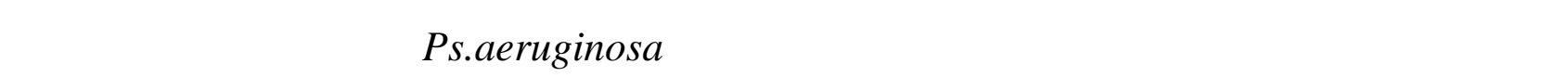

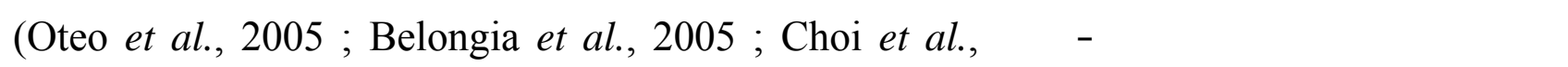

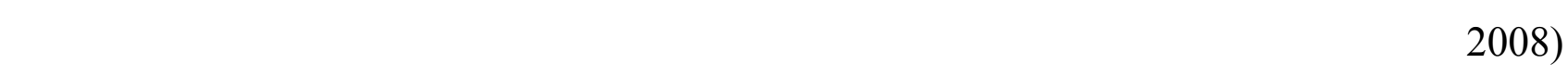

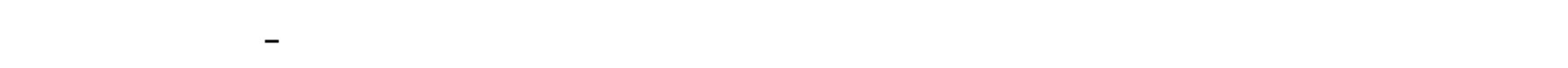

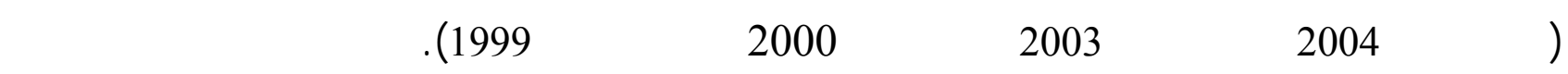

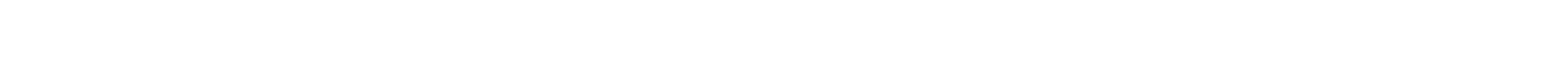

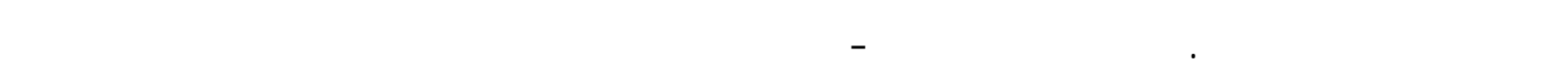

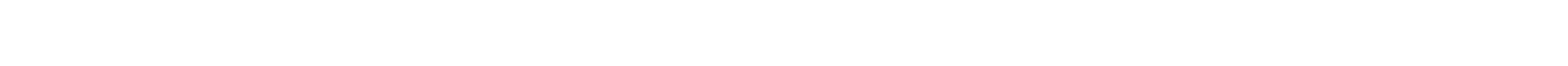

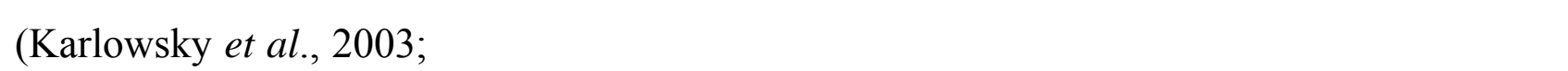
.Orrett, 2004; Jean et al., 2009)

\section{المصادر العربية}

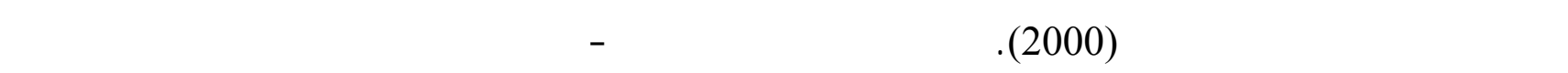

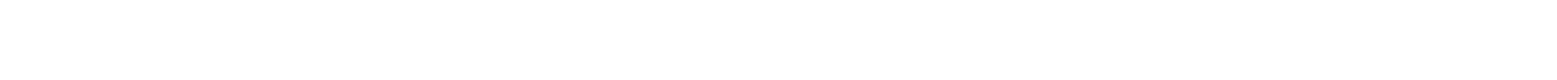
الجراثيم. بسالة ملجستير. كلية العلو، جلمعة الموصل، العراق.

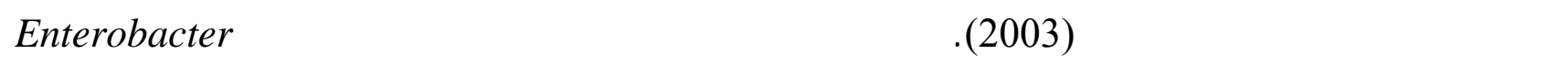
aerogenes ملجستير، كلية العلوم، جلمعة الموصل، العراق.

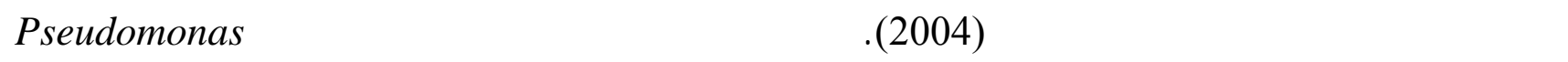
aeruginosa جلمعة الموصل، العراق.

المعاضيدي، جلسم فتحي علي (1999). دربلست على الأحياء المجهرية المسبة لالتهابِ لت الأنن الوس طى القيجي عند الألفل في مدينة الموصل. بسالة ملجستير، كلية العلوم، جلمعة الموصل، العراق.

\section{المصاكر الأجنبية}

Aminizadeh, Z.; Kashi, M. (2011). Prevalence of multi-drug resistance and pandrug resistance among multiple gram-negative species :experience in one teaching hospital, Tehran, Iran. Int. Res. J. Microbiol., 2(3), 90-95.

Bartlett, J.G.; Breiman, R.F.; Mandell, L.A.; File, T.M. (1998). Community-acquired pneumonia in adults: Guidelines for management. Clin. Infect. Dis., 26, 811-838. 
Belongia, E.A.; Knobloch, M.J.; Kieke, B.A.; Davis, J.; Janette, C.; Besser, R. (2005). Impact of statewide program to promote appropriate antimicrobial drug use. Emerg. Infect. Dis., 11, 912-920.

Blazquez, J.; Baquero, M.R.; Canton, R.; Alos, A.; Baquero, F. (1993). Characterization of a new TEM-type $\beta$-lactamase resistant to clavulinate, sulbactam and tazobactam in a clinical isolate of Escherichia coli . Antimicrob. Agents Chemother., 37, 20592063.

Bononi, I.; Balatti, V.; Gaeta, S.; Tgnon, M. (2008). Gram negative bacteria lipopolysaccharide retention a positively charged new generation filter. Appl. Environ. Microbiol., 74(20), 6470-6472.

Bradford, P.A. (2001). Extended-spectrum $\beta$-lactamases in the 21 st century: characterization, epidemiology, and detection of this important resistant threat. Clin. Microbiol. Rev., 14, 933-951.

Choi, S.H.; Lee, J.E.; Park, S.J.; Choi, S.J.; Lee, S.O.; Jeong, J.Y.; Kim, M.N.; Woo, J.H.; Kim, Y.S. (2008). Emergence of Antibiotic resistance during therapy for infection caused by Enterobacteriaceae producing AmpC $\beta$-lactamase: Implication for antibiotic use. Antimicrob. Agents Chemother. 52(3), 995-100.

Crofton, J.; Douglas, A. (1988). "Respiratory Diseases". 3rd ed., P.G. publishing pte. Ltd., Singapore. pp. 324-376.

Dugal, S.; Fernandes, A. (2011). Carbapenem hydrolyzing metallo- $\beta$-lactamases: a review. Int. J. Curr. Pharm. Res., 3(3), 9-16.

Forbes, B.A.; Sahm, D.F.; Weissfeld, A.S. (2007). "Bailly and Scott's Diagnostic Microbiology". 12th ed. Mosby company Inc., USA. pp. 256-277.

Gradelski, E.; Valera, L.; Bonner, D.; Fung-Tomc, J. (2001). Synergistic activities of gatifloxacin in combination with other antimicrobial agents against Pseudomonas aeruginosa and related species . Antimicrob. Agent Chemother., 45, 3220-3222.

Jean, S.S.; Hsueh, P.R.; Lee, W.S.; Chang, H.T.; Chon, M.Y.; Chen, I.S.; Lee, C.Y. (2009). Nationwide surveillance of antimicrobial resistance among non fermentative bacteria in intensive care units in Taiwan: SMART program data 2005. Int. J. Antimicrob. Agents, 33, 266-271.

Karlowsky, J.A.; Jones, M.E.; Thornsberry, C.; Friedland, I.R.; Sahm, D.F. (2003). Trends in antimicrobial susceptibilities among Enterobacteriaceae isolated from hospitalized patients in the United States from 1998 to 2001. Antimicrob. Agents Chemother., 47, 1672-1680.

Khan, J.A.; Iqbal, Z.; Rahman, S.U.; Farzana, K.; Khan, A. (2008). Prevalence and resistance pattern of Pseudomonas aeruginosa against antibiotics. Pak. $J$. Pharmacol. Sci., 21 (3), 311-315.

Livermore, D.M. (1995). $\beta$-lactamases in laboratory and clinical resistance. Clin. Microbiol. Rev., 8, 557-584.

Luzzaro, F.; Docquier, J.D.; Colinon, C.; Endimiani, A.; Lombardi, G.; Amicosante, G.; Rossolini, G.M.; Toniolo, A. (2004). Emergence in Klebsiella pneumoniae and Escherichia coli clinical isolates of the VIM-4 metallo- $\beta$-lactamase encoded by a conjugative plasmid. Antimicrob. Agent Chemother., 48, 648-650.

Mac Faddin, J.F. (1985). "Biochemical Tests for Identification of Medical Bacteria". 2nd ed., Williams and Wilkins company, Baltimore, U.S.A. pp. 114-388. 
Metlay, J.P.; Powers, J.H.; Dudley, M.N.; Christiansen, K.; Finch, R.G. (2006). Antimicrobial drug resistance, regulation, and research. Emerg. Infect. Dis., 12, 183-190.

Miriagou, V.; Tzelepi, E.; Gianneli, D.; Tzouvelekis, L.S. (2003). Escherichia coli with a self-transferable, multiresistant plasmid coding for metallo- $\beta$-lactamase VIM-1. Antimicrob. Agents Chemother., 47, 395-397.

National Committee for Clinical Laboratory Standards, NCCLS (2004). "Performance Standards for Antimicrobial Susceptibility Testing; 14 th Informational Supplement" . M100-S14,Vol. 24, No.1, NCCLS, Wayne, PA, U.S.A.

National Committee for Clinical Laboratory Standards, NCCLS (2003). "Performance Standards for Antimicrobial Disk Susceptibility Tests". Approved Standards-8th ed., M2-A8, Vol. 20, No.1, NCCLS, Wayne, PA, U.S.A.

Orrett, F.A. (2004). Antimicrobial susceptibility survey of Pseudomonas aeruginosa strains isolated from clinical sources. J. N. Med. Ass., 96, 1065-1069.

Oteo, J.; Lazaro, E.; Abajo, F.; Baquero, F.; Campos, J. (2005). Antimicro-bial-resistant invasive Escherichia coli, Spain . Emerg. Infect. Dis., 11, 546-553.

Patzer, J.A.; Dzierzanowska, D.; Turner, P.J. (2007). High activity of meropenem against gram negative bacteria from pediatric intensive care units, 2001-2005. Int. J. Antimicrob. Agents, 29, 285-288.

Saeed, A.; Khatoon, H.; Ansari, F.A. (2009). Multidrug resistant gram negative bacteria in clinical isolates from Karatchi. Pak. J. Pharm. Sci., 22(1), 44-48.

Schwalbe, R.; Moore, L.S.; Goodwing, A. (2007). "Antimicrobial Susceptibility Testing Protocols ". Taylor and Francis Group Inc., U.S.A. pp. 40-120.

Thomson, K.S.; Moland, E.S. (2001). Cefepime, piperacillin-tazobactam, and the inoculum effect in tests with extended-spectrum $\beta$-lactamase-producing Enterobacteriaceae . Antimicrob. Agents Chemother., 45(12), 3548-3554.

Varaiya, A.; Kulkarni, M.; Dorgra, J.(2008). Incidence of metallo $\beta$-lactamase producing Pseudomonas aeruginosa in ICU patients. Ind.J. Med.Res., 127, 398-402.

Wichard, J.M.; Joyce, K.; Fey, P.D.; Nelson, J.M.; Angulo, F.J.; Barrett, T.J. (2005). Blactam resistance and Enterobacteriaceae, United States. Emerg. Infect. Dis.,11, 1464-1466.

Winn, W.C.; Allen, S.D.; Janda, W.M.; Koneman, E.W.; Procop, G.W.; Schreckenberger, P.C.; Woods, G.L. (2006). "Koneman's Color Atlas and Text book of Diagnostic Microbiology". 6th ed., Lippincott Williams and Wilkins, U.S.A. pp. 212-278. 